Revista de Psicología de la PUCP. Vol. XXII, 2, 2004

\title{
Perspectiva temporal futura y satisfacción con la vida a lo largo del ciclo vital
}

\author{
Patricia Martínez U. ${ }^{1}$ \\ Pontificia Universidad Católica del Perú
}

Este estudio aborda los constructos de Perspectiva Temporal Futura, en sus diferentes dimensiones (Metas, ubicación temporal de las metas y Actitudes Temporales) y de Satisfacción con la vida, desde la adolescencia en adelante. A través de un diseño no experimental, evolutivo transversal, se evaluó a 570 personas de ambos sexos entre los 16 y los 65 años, de dos niveles socioeconómicos de Lima, Perú. Los instrumentos utilizados fueron el Método de Inducción Motivacional (MIM), la Escala de Actitudes Temporales (TAS) de Nuttin (1985) y la Escala de Satisfacción con la Vida (SWLS) de Pavot y Diener (1993). Los resultados muestran que la edad supone diferencias importantes en la construcción de las metas y su ubicación temporal, así como en las actitudes hacia el presente, pasado y futuro y en los niveles de satisfacción. Asimismo, evidencia que el nivel socioeconómico, más que el sexo, influye en estos procesos.

Palabras clave: perspectiva temporal futura, satisfacción con la vida, ciclo vital.

\section{Future time perspective and satisfaction with life across life span}

The present research studies Satisfaction with Life and Future Time Perspective, in its different dimensions (goals, temporal location of goals and temporal attitudes), from adolescence to elder ages. This study evaluated 570 males and females between 16 and 65 years old, from two different socioeconomic levels in Lima, Peru, through a nonexperimental, evolutionary transversal design. Tools used in this research were the Motivational Induction Method (MIM) and the Temporal Attitudes Scale (TAS) by Nuttin (1985), and the Satisfaction with Life Scale (SWLS) by Pavot \& Diener (1993). Results show that there are significant differences on the construction and temporal location of goals, attitudes towards present, past and future and in the levels of Life Satisfaction due to the variable age. It was also found that socioeconomic level influences these processes more than gender.

Key words: future time perspective, satisfaction with life, life span. Asociada del Departamento de Psicología y Coordinadora de la Maestría en Estudios Teóricos en Psicoanálisis, Pontificia Universidad Católica del Perú. Correo electrónico: pmartin@pucp.edu.pe 

Lo que somos en el presente es en gran parte producto de nuestra experiencia pasada y de aquello que queremos llegar a ser en el futuro. La imagen que tenemos de nosotros mismos en el futuro actúa como un modelo que guía nuestra conducta y que dirige nuestros esfuerzos hacia un fin determinado. Resulta así evidente que la conducta posee una dimensión temporal. Diversos autores desde la psicología han dedicado sus investigaciones a tratar de entender cómo se articulan el pasado, presente y futuro en la conducta humana. Entre ellos destacan los aportes de Lens (1994, 1998), Nurmi $(1991,1994)$, Nuttin $(1982,1985)$ y Zaleski (1994), entre otros. Cada uno de ellos ha realizado un conjunto de investigaciones enfatizando diferentes aspectos de la conducta y su relación con aspectos temporales.

En la presente investigación tomaremos como punto de partida la teoría de Nuttin, para complementarla con los aportes de otros investigadores. Para Nuttin (1982) lo que determina la influencia del ambiente sobre la conducta no es la existencia real de un hecho, sino su significación para el individuo. En la conducta influye tanto el proceso de aprendizaje anterior como el proyecto futuro; los actos se convierten así en actos instrumentales con relación a un fin. El individuo elabora proyectos que concretan sus necesidades para luego actuar en el presente y buscar maneras de satisfacer esas necesidades.

Se puede hablar, de este modo, de una estructura jerárquica de fines o metas que van otorgando un significado a cada uno de los actos que el individuo realiza. Es posible hablar de una línea de desarrollo representada por la formación de proyectos, cuya dinámica se basa en la tendencia humana a sobrepasar el estado logrado, es decir, al desarrollo personal. La necesidad pone en marcha la actividad cognitiva a través de la manipulación mental de 
objetos y de las vías disponibles o imaginables de satisfacer la necesidad; es, asimismo, la que impulsa al organismo a alcanzarlos.

La conducta del individuo se regula en última instancia de acuerdo con los proyectos que se coloca a sí mismo y que reflejan una concepción de sí. Desarrollarse, en la perspectiva de Nuttin, viene a ser transformarse en lo que se desea ser, y se constituye en un aspecto central del desarrollo humano.

\section{Perspectiva temporal futura (PTF)}

Para Nuttin (1985), la perspectiva temporal futura supone la representación de objetos localizados en el futuro e incluye dos aspectos: los objetos motivacionales o metas, que constituyen el contenido de la PTF, y la ubicación temporal de esas metas, que constituye su extensión. Asimismo, las actitudes hacia el presente, pasado y futuro constituyen lo que el autor define como actitudes temporales, también asociadas a la PTF. La noción de perspectiva temporal se encuentra, para Nuttin, a la base de la riqueza y variedad de actividades que una persona se propone. En este contexto, es de vital importancia reconocer el papel que desempeñan las condiciones de vida y experiencias personales en la construcción de la perspectiva temporal. Nurmi (1994) reporta una serie de investigaciones en las cuales encuentra que las metas están relacionadas a las tareas de desarrollo propias de cada etapa evolutiva.

La localización precisa de un evento en el futuro resulta de la experiencia individual con el medio. Para Zaleski (1994), las personas con una larga PTF son más persistentes en su intento de alcanzar la meta y obtienen mayor satisfacción de las acciones que se dirigen hacia la meta.

El contenido de la PTF está constituido entonces por eventos, situaciones y proyectos que se encuentran en la mente de las personas. Estos tienen además un afecto positivo o negativo para 
Perspectiva temporal futura y satisfacción con la vida a lo largo del ciclo vital

cada persona. Este afecto placentero o displacentero, referido al pasado, al presente o al futuro, constituye una actitud afectiva. Estas actitudes son importantes porque tienen un impacto en la motivación presente y, por lo tanto, en la conducta (Nuttin, 1985). Para Zaleski (1994), el futuro es concebido como un espacio temporal abierto que evoca actitudes positivas y negativas, es un espacio de planeamiento y realización de metas, por lo que tiene un poder motivacional. Cada una de estas actitudes puede predominar en un período de tiempo y puede ser también relativamente estable.

El concepto de actitud temporal contiene una fuerte carga afectiva o emocional. Las personas pueden tener una actitud negativa hacia el pasado y presente pero positiva u optimista hacia el futuro, sin embargo, por lo general es más común encontrar una alta correlación entre las tres (Van Calster, Lens \& Nuttin, 1987).

\section{Satisfacción con la vida}

La satisfacción con la vida corresponde al componente cognitivo del bienestar subjetivo. Se refiere al proceso en el que las personas evalúan la calidad de sus vidas de acuerdo a su propio criterio y de manera global (Pavot \& Diener, 1993).

Se han realizado numerosos estudios sobre la satisfacción con la vida, asociándola a factores externos como la salud, el grado de instrucción, el estado civil o el nivel de ingresos, entre otros. En la mayor parte de estos estudios se ha encontrado una asociación débil, por lo que la investigación actual tiende a centrarse más bien en variables internas o de personalidad. Se han encontrado así correlaciones importantes con extroversión y neuroticismo, de lo que se deduce que existe una interacción entre personalidad y situación asociada a los niveles de satisfacción (Diener, Oishi \& Lucas, 2003). 
En otras palabras, podría hablarse de dos maneras de entender la satisfacción: como el resultado de un conjunto de experiencias positivas, o como una disposición general de la personalidad. Así, unos la consideran como producto de variables de personalidad y temperamento, y otros la consideran como algo más bien sensible a las circunstancias. Evidentemente, existen también estudios que conectan ambos aspectos.

\section{Perspectiva temporal y ciclo vital}

A partir de lo descrito hasta aquí podemos suponer que en cada etapa del desarrollo el tipo de metas que cada persona construye tendrá características distintas de acuerdo a las tareas de desarrollo que le son propias, y que las vivencias del pasado, presente y futuro serán diferentes de acuerdo a la importancia que se otorgue a cada periodo temporal. Asimismo, el modo en que las personas elaboran o construyen sus proyectos estaría además directamente relacionado con los diferentes niveles de bienestar o satisfacción con la vida. En este contexto, nos interesa especialmente el desarrollo desde la adolescencia y, en particular, desde la adolescencia tardía hasta la tercera edad o adultez tardía.

La adolescencia tardía, considerada generalmente como la etapa de tránsito a la etapa adulta, se ubica básicamente en la finalización de los estudios escolares y el inicio de los estudios superiores. La tarea primordial del adolescente es lograr una definición de sí mismo y de lo que quiere llegar a ser en un futuro (Rice, 2000). Esta es una etapa de cambios en la cual el adolescente tiene como tarea central definir su identidad y con ello el rumbo de su vida (Erikson, 1993).

La etapa adulta, por otro lado, supone también un conjunto de tareas que varían de acuerdo a la edad. El crecimiento adulto se define a partir de ciertos logros sociales, muy influidos también por la cultura (Craig, 1994). En general existe un consenso en 
Perspectiva temporal futura y satisfacción con la vida a lo largo del ciclo vital

considerar tres etapas básicas en el desarrollo adulto, aunque con ciertas discrepancias con respecto a la duración de cada una de ellas y a su denominación. Así, la adultez temprana o juventud se extiende de los 20 a los 40 años aproximadamente, la adultez media o madurez de los 40 a los 60 y la adultez tardía o vejez de los 60 o 65 en adelante (Craig, 1994; Lefrancois, 2001; StassenBerger, 2001).

Los inicios de la adultez temprana generalmente se asocian al logro de la independencia, tanto económica como emocional, lo que supone la inserción en el mundo laboral. De este modo el trabajo resulta ser uno de los ejes fundamentales de desarrollo; el otro eje lo constituyen los vínculos que el adulto establece con los demás. Para Neugarten (1999), uno de los objetivos más importantes de esta etapa es conseguir un equilibrio entre el situarse y prosperar, cumplir con las nuevas obligaciones, establecer vínculos más profundos y conseguir reconocimiento.

La adultez media corresponde a una de las etapas más productivas de la vida, tanto en el ámbito laboral como en el social. Las tareas fundamentales de esta etapa tienen que ver con la perpetuación de la cultura y la aceptación de los cambios físicos. Esta es la etapa que muchos describen como la de la crisis media de la vida, donde surge una suerte de evaluación de todo lo pasado; la percepción y valoración del tiempo presenta así cambios importantes con respecto a la etapa anterior (Stassen-Berger, 2001). Aquí aparecen los primeros signos de envejecimiento y la necesidad de adaptarse a ellos (Craig, 1994).

Finalmente la adultez tardía se encuentra asociada al envejecimiento físico que resulta de una combinación de factores genéticos y ambientales (Craig, 1994). Son muchos los prejuicios y estereotipos sobre la vejez. Para Neugarten (1999), se puede hablar de viejos-jóvenes para referirse a las personas entre los 55 y 85 años con buena salud, bien integrados a la vida familiar y a la comunidad e incluso políticamente activos. Como contraste, los viejos-viejos, mayores de 85 años, son más vulnerables y requieren de cuidado y ayuda especial. 
En esta etapa, las personas se vuelven más reflexivas y filosóficas, evalúan su vida, sus éxitos y fracasos. Se presenta una revisión general de la vida, comparando el pasado y el presente. Se considera una etapa de sabiduría que se asocia a que los procesos mentales en la vejez pueden ser adaptables y creativos (Stassen-Berger, 2001). Los temas centrales de la adultez tardía giran en torno a la salud y al establecimiento de vínculos sociales. El proceso de envejecimiento y el acercamiento al final de la vida marcan la dinámica de esta etapa, muy influida por el estado de salud general y por factores sociales.

Existe una cantidad considerable de investigaciones que vinculan la PTF a diferentes momentos del ciclo vital; entre ellas se encuentran las de Bouffard, Bastin y Lapierre (1994), Díaz Morales y Sánchez López (2002), Frazier, Johnson, Gonzales y Kafka (2002), Herrera (2002), Husman y Lens (1999), Lennings (2000), Martínez (2002), Nurmi (1991, 1994), Nurmi, Poole y Seginer (1992, 1995), Salmela y Nurmi (1997), Staudinger, Bluck y Herzberg (2003), y De Volder y Lens (1982).

En base a lo anteriormente expuesto, la presente investigación intenta abordar el constructo de perspectiva temporal futura en sus diferentes dimensiones (metas, ubicación temporal de las metas y actitudes temporales) y el de satisfacción con la vida, en hombres y mujeres que se encuentran en diferentes etapas de desarrollo y que pertenecen a diferentes niveles socioeconómicos.

Los objetivos son:

- Describir y comparar los diferentes aspectos de la perspectiva temporal futura (metas, extensión y actitudes temporales) en personas que se encuentran en diferentes etapas evolutivas.

- Describir y comparar la satisfacción con la vida en cada uno de los grupos etáreos.

- Establecer asociaciones entre las diferentes variables del estudio. 
Perspectiva temporal futura y satisfacción con la vida a lo largo del ciclo vital

Asimismo, se intenta analizar en qué medida estas variables pueden verse influidas por variables como el sexo y el nivel socioeconómico.

\section{Metodología}

Se trata de un estudio no experimental de tipo evolutivo transversal, en el que se evaluaron grupos de distintas edades en un mismo momento, con el fin de compararlos y analizar las características de la PTF propias de cada etapa evolutiva (Hernández, Fernández \& Baptista, 1998).

\section{Participantes}

La muestra total del presente estudio estuvo conformada por 570 personas de ambos sexos comprendidas entre los 16 y los 65 años de edad, pertenecientes a dos sectores socioeconómicos (Cuadro 1). Se trabajó con cuatro grupos de edad: adolescencia tardía (16 a 18 años), que corresponde al final de la etapa escolar; el periodo de $\operatorname{los} 22$ a los 25 años, momento en el que por lo general los jóvenes se encuentran culminando su formación profesional e ingresando a la adultez temprana; el periodo de los 40 a los 45 años, que constituiría el inicio de la adultez media; y, de los 60 a los 65 años, que corresponde al inicio de la adultez tardía (Craig, 1994; Lefrancois, 2001; Stassen-Berger, 2001). 
Cuadro 1

Distribución de la muestra

\begin{tabular}{cccccc}
\hline \multirow{2}{*}{ Edad } & \multicolumn{2}{c}{ Hombres } & \multicolumn{2}{c}{ Mujeres } & \multirow{2}{*}{ Total } \\
\cline { 2 - 5 } & NSE Alto & NSE Bajo & NSE Alto & NSE Bajo & \\
\hline $16-18$ & 36 & 36 & 35 & 36 & 143 \\
$22-25$ & 36 & 35 & 35 & 36 & 142 \\
$40-45$ & 37 & 35 & 35 & 36 & 143 \\
$60-65$ & 35 & 35 & 36 & 36 & 142 \\
Total & 144 & 141 & 141 & 144 & 570 \\
\hline
\end{tabular}

La definición del nivel socioeconómico se realizó en base a tres criterios: el tipo de centro de estudios (para adolescentes y adultos jóvenes) o el tipo de actividad que desempeña el participante (para adultos medios y tardíos), el nivel de instrucción de los padres (para adolescentes y adultos jóvenes) o del participante (para adultos medios y tardíos) y el distrito de residencia. A partir de ellos se definieron dos grupos: uno de nivel alto y medio alto que corresponde a los niveles $\mathrm{A}$ y $\mathrm{B}$, y otro de nivel bajo y medio bajo que corresponde a los grupos $\mathrm{C}$ y $\mathrm{D}$ (Apoyo, 2002).

Tomando en cuenta todos estos criterios se realizó un muestreo no probabilístico por cuotas (Hernández, Fernández \& Baptista, 1998) con el objetivo de cubrir un mínimo de 30 casos para cada grupo de edad, sexo y nivel socioeconómico.

\section{Instrumentos}

Método de Inducción Motivacional (MIM)

Se trata de una prueba orientada a identificar las metas $u$ objetos motivacionales propios de cada individuo y situarlos en una escala temporal. La prueba consiste en un cuadernillo con 
Perspectiva temporal futura y satisfacción con la vida a lo largo del ciclo vital

tarjetas que contienen frases en primera persona que inducen al examinado a expresar sus metas, deseos y temores. A partir de ello se pueden identificar categorías motivacionales a las cuales pertenece cada una de estas metas. Se utilizó la forma abreviada del MIM que comprende 30 inductores, 20 positivos y 10 negativos. La codificación de la prueba se realizó a partir de los códigos desarrollados por Nuttin y Lens (en Nuttin, 1985), que incluyen el análisis de contenido y los códigos temporales.

Para el análisis de contenido se proponen diez categorías generales y un conjunto de subcategorías. En este caso se trabajó con todas las categorías y subcategorías propuestas por los autores $y$, posteriormente, se eliminaron las que no alcanzaron una presencia significativa. Las categorías y subcategorías que se usaron en el presente estudio fueron:

- Sí Mismo (S): metas relativas a la personalidad. Comprende: Autoconcepto $\left(\mathrm{S}_{\mathrm{c}}\right)$, Preservación $\left(\mathrm{S}_{\mathrm{pre}}\right)$, Autonomía $\left(\mathrm{S}_{\mathrm{aut}}\right)$, Habilidades $\left(\mathrm{S}_{\text {apt }}\right)$ y Rasgos de Carácter $\left(\mathrm{S}_{\text {car }}\right)$.

- Autorrealización (SR): desarrollo del sí mismo. Comprende: Área Profesional $\left(\mathrm{SR}_{2}\right)$ y Ámbito Estudiantil $\left(\mathrm{SR}_{3}\right)$.

- Realización ( $\mathrm{R})$ : formas de actividad productiva. Comprende: Actividades Profesionales $\left(R_{2}\right)$ y Actividades Estudiantiles $\left(\mathrm{R}_{3}\right)$.

- Contacto (C): interacción con otros. Comprende: necesidad de ser contactado por otros $\left(C_{2}\right)$, Deseos para Otros $\left(C_{3}\right)$, Deseos Humanitarios $\left(\mathrm{C}_{3} \mathrm{H}\right)$, Contacto Íntimo $\left(\mathrm{C}_{\text {int }}\right)$, Contacto Altruista $\left(\mathrm{C}_{\mathrm{att}}\right)$ y Contacto Egocéntrico $\left(\mathrm{C}_{\mathrm{eg}}\right)$. Además se incluye el tipo de contacto: familiar, parental, filial, de pareja, amical y social.

- Exploración (E): motivaciones cognitivas, de conocimiento.

- Trascendencia (T): metas de tipo religioso o existencial.

- Posesiones (P): deseo de poseer o adquirir objetos.

- Diversión (L): actividades de recreación o placer. 
Del mismo modo, del conjunto de códigos temporales que proponen Nuttin y Lens (Nuttin, 1985) se trabajó con cuatro categorías temporales:

- Futuro cercano: menos de 2 años.

- Futuro distante: más de 2 años.

- Presente abierto: de ahora en adelante.

- Pasado: referencias al pasado.

Nuttin (1985) reporta diferentes estudios sobre la validez y confiabilidad de la prueba que confirman su sensibilidad para identificar los objetos motivacionales de grupos específicos y una alta consistencia entre evaluadores. En el Perú se han realizado dos estudios con el MIM en adolescentes escolares en los que se confirma la utilidad de la prueba para la medición de la perspectiva temporal futura (Herrera, 2002; Martínez, 2002). Para la presente investigación se obtuvo un coeficiente intracodificador de .86 para los contenidos y .93 para los códigos temporales.

Escala de Actitudes Temporales (TAS)

La escala fue construida por Nuttin con el fin de evaluar la actitud hacia el pasado, presente y futuro. Esta escala está basada en la técnica del diferencial semántico y está compuesta por 19 pares de adjetivos para la evaluación de la actitud hacia el futuro y el presente y por 15 pares para la evaluación de la actitud hacia el pasado. Las personas evaluadas deben ubicarse en cada uno de los pares de acuerdo a una escala de 7 puntos, donde 7 representa el extremo positivo y 1 el negativo (Nuttin, 1985).

El puntaje total de la prueba para cada una de las dimensiones temporales (pasado, presente y futuro) representa una actitud general que se analiza en base a tres categorías: una actitud positiva se encuentra en puntajes superiores a 4.49 , una 
Perspectiva temporal futura y satisfacción con la vida a lo largo del ciclo vital

actitud neutral supone puntajes entre 3.5 y 4.49 , y una actitud negativa se observa en puntajes inferiores a 3.5 .

Del mismo modo que el MIM, la escala en su versión de actitud hacia el futuro fue probada en una investigación previa con adolescentes de Lima (Martínez 2002), demostrando una elevada consistencia interna (.83). En la presente investigación se realizó una correlación ítem-test mediante el coeficiente alfa de Cronbach. Todos los ítems presentaron una correlación positiva; la forma Presente alcanzó un alfa total de .847; la forma Futuro, de .893 ; y, la forma Pasado, de .805 .

Escala de Satisfacción con la Vida (SWLS)

Se trata de una escala destinada a evaluar la satisfacción global con la vida, desarrollada por Pavot y Diener (1993). Consiste en un conjunto de cinco afirmaciones frente a las cuales las personas deben indicar su nivel de acuerdo o desacuerdo en una escala de 7 puntos. El puntaje total de la prueba oscila entre 5 y 35; un puntaje de 20 representa un punto neutral e indica que estas personas no están ni satisfechas ni insatisfechas con su vida; del 21 al 25 están ligeramente satisfechas; del 15 al 19 ligeramente insatisfechas; del 26 al 30 satisfechas; y, del 10 al 14 insatisfechas.

Los estudios normativos realizados con la prueba en diferentes grupos y nacionalidades (EE.UU., Francia, China, Rusia, etc.) indican que las medias en todos estos estudios con poblaciones normales se ubican en la categoría de ligeramente satisfechos o satisfechos. En muestras clínicas, sin embargo, los puntajes tienden a ser menores (Pavot \& Diener, 1993), lo que constituye una prueba de su validez.

De acuerdo a los autores, numerosos estudios con la prueba demuestran una alta consistencia interna (alfa de .87) y una estabilidad temporal moderada. El análisis factorial de la prueba 
muestra la presencia de un solo factor que explica el $66 \%$ de la varianza, que además se mantiene en diferentes estudios. Para el presente estudio se realizó un análisis factorial de la prueba que confirma los estudios reportados. Se encontró un solo factor que explicaba el $57.63 \%$ de la varianza total. En el análisis de la consistencia interna de la prueba mediante el alfa de Cronbach se encontró un alfa total de .81 .

\section{Procedimiento}

La selección de los participantes se realizó de diferentes maneras de acuerdo al grupo etáreo. El grupo de adolescentes se obtuvo de colegios particulares y nacionales de Lima, el grupo de adultos jóvenes se obtuvo de universidades privadas y nacionales, y los grupos de adultos medios y tardíos se obtuvieron a través de centros de trabajo como pequeñas empresas, locales comerciales o centros de salud.

Una vez que se completó la cantidad de casos proyectada, se procedió a eliminar a todos aquellos casos que no cumplieran con los tres criterios propuestos para la definición del nivel socioeconómico, que tuvieran más de tres respuestas en blanco en el MIM, más de dos ítems en blanco en el TAS o un ítem en blanco en el SWLS.

Una vez recogidos todos los datos se aplicó la Prueba de Bondad de Ajuste de Kolmogorof Smirnov para determinar la normalidad de las puntuaciones de todas las variables del estudio. Ninguna de las variables presentó una distribución normal, por lo que se optó por usar estadísticos no paramétricos. Así, para la comparación de variables por grupos de edad se usó la prueba de Kruskal Wallis con un $p<.05$. Adicionalmente se usó el criterio de Cohen para determinar el tamaño de las diferencias entre grupos. Para las comparaciones por sexo y nivel socioeconómico se utilizó la $U$ de Mann Whitney. 
Las correlaciones entre variables se realizaron mediante el coeficiente $r$ de Pearson, nuevamente con el criterio de Cohen para establecer la significación de las mismas. Finalmente se realizó un análisis de conglomerados con todas las variables del estudio, con el fin de determinar posibles agrupaciones entre los participantes, para ello se utilizó el análisis de clusters mediante el Método Ward y se aplicó un análisis de varianza (ANOVA) para determinar las variables que presentaban diferencias significativas entre clusters, además del criterio de diferencias de Cohen. Finalmente se realizó la prueba de Chi Cuadrado para determinar diferencias entre grupos de edad, sexo y nivel socioeconómico.

\section{Resultados}

\section{Perspectiva temporal futura}

El análisis de la perspectiva temporal futura supone dos aspectos: las metas u objetos motivacionales, y la extensión de las metas o su ubicación temporal.

\section{Metas}

En la Figura 1 se presentan las metas del grupo total. Aquí se puede observar que las metas que mayor frecuencia presentan son las relacionadas a contacto interpersonal, sí mismo y autorrealización. 
Patricia Martínez U.

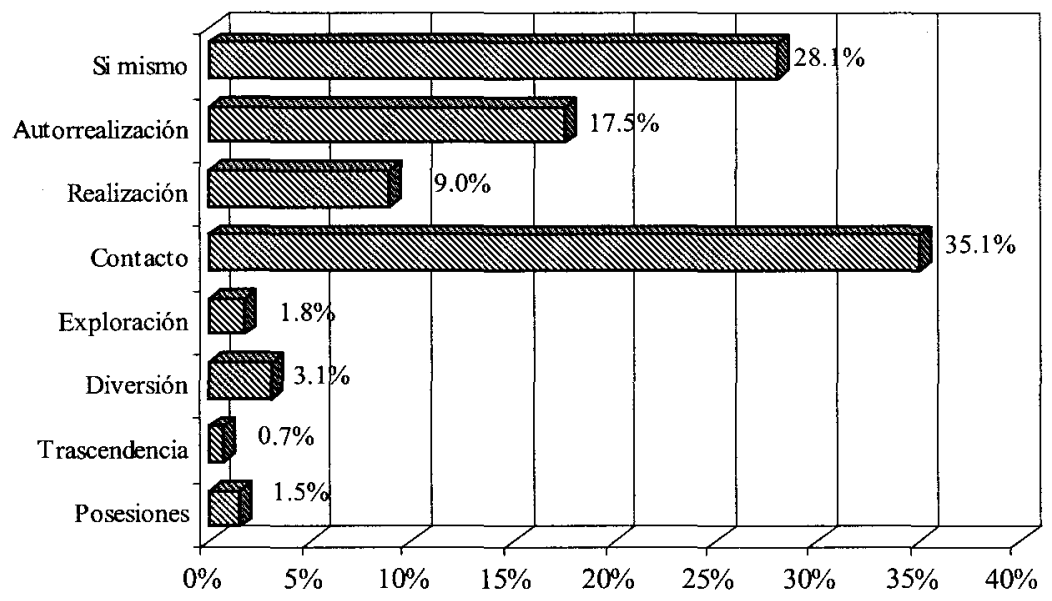

Figura 1. MIM: Metas del grupo total.

La categoría más frecuente es la de Contacto Interpersonal, donde encontramos diferencias estadísticamente significativas de acuerdo al grupo de edad. Aquí se puede observar (Cuadro 1) que no aparece mayor diferencia entre los adolescentes y adultos jóvenes por un lado, y entre los adultos medios y tardíos por el otro, pero sí una clara diferencia entre los menores (16-18 y 2225) y los mayores (40-45 y 60-65), que son los que presentan mayor cantidad de metas vinculadas al Contacto Interpersonal, por lo que se puede inferir que el vínculo interpersonal adquiere mayor importancia con la edad. 
Perspectiva temporal futura y satisfacción con la vida a lo largo del ciclo vital

Cuadro 1

Diferencias significativas en contacto interpersonal por grupo de edad

\begin{tabular}{lccccccccc}
\hline Categoria & \multicolumn{2}{c}{$16-18$} & \multicolumn{2}{c}{$22-25$} & \multicolumn{2}{c}{$40-45$} & \multicolumn{2}{c}{$60-65$} & \multirow{2}{*}{$K-W$} \\
\cline { 2 - 8 } & $M$ & $D E$ & $M$ & $D E$ & $M$ & $D E$ & $M$ & $D E$ & \\
\hline Contacto & 8.83 & 4.15 & 8.65 & 4.14 & $\mathbf{1 2 . 0 1}$ & 4.95 & $\mathbf{1 2 . 6 8}$ & 4.54 & $79.763^{* * *}$ \\
\hline$C_{3}$ & 2.84 & 2.70 & 2.42 & 2.38 & $\mathbf{6 . 2 9}$ & 3.68 & $\mathbf{5 . 5 9}$ & 3.72 & $132.496^{* * *}$ \\
$C_{3} H$ & 0.53 & 1.14 & 0.69 & 1.19 & $\mathbf{1 . 3 5}$ & 2.05 & $\mathbf{1 . 1 6}$ & 1.70 & $20.703^{* * *}$ \\
$C_{\text {int }}$ & $\mathbf{1 . 2 6}$ & 1.66 & $\mathbf{1 . 4 1}$ & $\mathbf{1 . 8 0}$ & 0.49 & 0.91 & 0.46 & $\mathbf{1 . 2 0}$ & $61.837^{* * *}$ \\
$C_{\text {eg }}$ & 0.11 & 0.34 & $\mathbf{0 . 2 6}$ & 0.57 & 0.08 & 0.27 & 0.08 & 0.37 & $17.850^{* * *}$ \\
Fam & 1.41 & 1.69 & 1.09 & 1.44 & $\mathbf{2 . 3 5}$ & 2.45 & $\mathbf{2 . 6 2}$ & 2.25 & $57.116^{* * *}$ \\
Hijos & 0.03 & 0.17 & 0.11 & 0.35 & $\mathbf{2 . 7 7}$ & 2.81 & $\mathbf{2 . 5 1}$ & 3.06 & $250.668^{* * *}$ \\
Padr & $\mathbf{1 . 4 9}$ & 1.97 & 0.53 & 0.90 & 0.20 & 0.57 & 0.10 & 0.42 & $109.449^{* * *}$ \\
Amig & $\mathbf{0 . 5 0}$ & 0.88 & 0.25 & 0.57 & 0.18 & 0.48 & 0.08 & 0.29 & $41.190^{* * *}$ \\
Parej & $\mathbf{1 . 2 0}$ & 1.75 & $\mathbf{1 . 1 3}$ & 1.78 & $\mathbf{0 . 5 6}$ & 1.14 & 0.52 & 1.18 & $26.693^{* * *}$ \\
Socie & $\mathbf{0 . 3 2}$ & $\mathbf{1 . 1 9}$ & 0.56 & 1.40 & $\mathbf{1 . 0 7}$ & 2.19 & $\mathbf{0 . 9 6}$ & 1.86 & $33.688^{* * *}$ \\
\hline
\end{tabular}

$* * * p<.001$

Al interior de la categoría de Contacto encontramos diferencias significativas en Deseos para Otros $\left(C_{3}\right)$ y Deseos para la Humanidad $\left(\mathrm{C}_{3} H\right)$, donde destacan los grupos mayores; en Contacto Intimo $\left(C_{i n t}\right)$ predominan los menores y en Contacto Egocéntrico $\left(C_{e g}\right)$ es el grupo de adultos jóvenes el que se diferencia de los otros tres.

Al analizar el tipo de contacto, también se encuentran diferencias estadísticamente significativas en las seis categorías registradas. En Familia, Hijos y Sociedad los grupos mayores destacan significativamente sobre los menores. En Pareja, se da lo contrario, son los menores los que destacan sobre los mayores. En Padres y Amigos, las diferencias se presentan entre todos los grupos denotándose una tendencia a disminuir conforme aumenta la edad.

La segunda categoría en términos de frecuencia es la de las metas localizadas en el Sí Mismo, donde también encontramos 
diferencias estadísticamente significativas de acuerdo al grupo de edad, como se puede observar en el Cuadro 2. Aquí es el grupo de adultos jóvenes el que se distingue de los otros tres grupos, al presentar mayor cantidad de metas localizadas en sí mismo. También encontramos diferencias importantes en las subcategorías de Contenido, donde se puede ver que, si bien no existe mayor diferencia entre los adolescentes y los adultos jóvenes, estas subcategorías tienden a modificarse con la edad.

\section{Cuadro 2}

Diferencias significativas en Sí Mismo de acuerdo a grupo de edad

\begin{tabular}{lrrrrrrrrrr}
\hline Categoría & \multicolumn{2}{c}{$16-18$} & \multicolumn{2}{c}{$22-25$} & \multicolumn{2}{c}{$40-45$} & \multicolumn{2}{c}{$60-65$} & \multirow{2}{*}{$K-W$} \\
\cline { 2 - 8 } & $M$ & $D E$ & $M$ & $D E$ & $M$ & $D E$ & $M$ & $D E$ & \\
\hline Sí Mismo & 8.56 & 3.75 & $\mathbf{9 . 1 7}$ & 4.09 & 7.90 & 3.16 & 8.08 & 3.67 & $9.215^{*}$ \\
\hline$S_{c}$ & $\mathbf{0 . 4 5}$ & 0.71 & $\mathbf{0 . 4 2}$ & 0.79 & 0.24 & 0.56 & 0.11 & 0.35 & $28.718^{* * *}$ \\
$S_{p r e}$ & 0.42 & 0.83 & 0.58 & 0.86 & 1.03 & 1.32 & $\mathbf{1 . 7 4}$ & 1.70 & $84.329^{* * *}$ \\
$S_{\text {aut }}$ & $\mathbf{0 . 7 8}$ & 1.02 & $\mathbf{0 . 9 1}$ & 1.25 & 0.36 & 0.79 & 0.23 & 0.54 & $56.920^{* * *}$ \\
$S_{\text {apt }}$ & 0.33 & 0.59 & 0.31 & 0.55 & $\mathbf{0 . 5 5}$ & 0.89 & 0.27 & 0.56 & $10.780^{*}$ \\
\hline
\end{tabular}

${ }^{*} \mathrm{p}<.05,{ }^{* * *} \mathrm{p}<.001$

Las respuestas vinculadas al Autoconcepto y Autonomía tienden a disminuir con la edad. Las de Preservación, en cambio, tienden a aumentar con la edad. Un dato particular en esta subcategoría se encuentra en los diferentes tipos de preservación, donde encontramos que Preservación Física constituye el 64\% de este tipo de respuestas, de las cuales el $26 \%$ se encuentra en los adultos medios y el $62 \%$ en los adultos tardíos. En Aptitudes, las diferencias se ubican básicamente en el grupo de adultos medios que destaca sobre los otros grupos.

Con respecto a las categorías de Autorrealización, que se refiere a la búsqueda de realización personal a través de 
Perspectiva temporal futura y satisfacción con la vida a lo largo del ciclo vital

actividades profesionales o del estudio, y Realización, relativa a logros concretos en ambos tipos de actividades, encontramos que ambas presentan diferencias significativas por grupos de edad (Cuadro 3). En Autorrealización puede observarse que, si bien no existen mayores diferencias entre los adolescentes y los adultos jóvenes, aparece una clara tendencia a disminuir con la edad. En Realización se presenta la misma tendencia, aunque aquí es entre los adultos medios y tardíos donde no se encuentran mayores diferencias.

Para el análisis de las subcategorías cabe mencionar que, en el caso de Autorrealización, aparece un $81 \%$ de respuestas sin ninguna especificación (trabajo o estudios); este tipo de respuestas se presenta en un $58 \%$ en el grupo de adolescentes, $23 \%$ en los adultos jóvenes y en un $19 \%$ en los adultos medios y tardíos. Ambas categorías presentan diferencias significativas en sus respectivas subcategorías. Autorrealización Profesional $\left(S R_{2}\right)$ destaca especialmente en los adultos jóvenes, mientras que la Realización Profesional $\left(R_{2}\right)$ destaca en los adultos tardíos. Por otro lado, el interés en los estudios $\left(S R_{3}\right.$ y $\left.R_{3}\right)$ tiende a disminuir con la edad, tanto en Autorrealización como en Realización.

\section{Cuadro 3}

Diferencias significativas en Autorrealización y Realización por grupo de edad

\begin{tabular}{lccccccccr}
\hline Categoria & \multicolumn{2}{c}{$16-18$} & \multicolumn{2}{c}{$22-25$} & \multicolumn{2}{c}{$40-45$} & \multicolumn{2}{c}{$60-65$} & \multirow{2}{*}{$K-W$} \\
\cline { 2 - 8 } & $M$ & $D E$ & $M$ & $D E$ & $M$ & $D E$ & $M$ & $D E$ & \\
\hline Autorrealiz. & $\mathbf{5 . 9 9}$ & 3.52 & $\mathbf{6 . 3 2}$ & 3.46 & 4.87 & 3.66 & 3.79 & 3.25 & $51.445^{* * *}$ \\
$S R_{2}$ & 0.83 & 1.40 & $\mathbf{1 . 1 1}$ & 1.44 & 0.73 & 1.29 & 0.76 & 1.43 & $14.771^{* *}$ \\
$S R_{3}$ & $\mathbf{0 . 2 4}$ & 0.69 & 0.17 & 0.39 & 0.08 & 0.39 & 0.03 & 0.17 & $22.686^{* * *}$ \\
\hline Realización & $\mathbf{4 . 1 9}$ & 3.28 & 2.75 & 2.58 & 1.85 & 2.09 & 1.99 & 2.07 & $64.889^{* * *}$ \\
$R_{2}$ & 1.15 & 1.36 & 0.88 & 1.49 & 1.19 & 1.76 & $\mathbf{1 . 5 0}$ & 1.97 & $10.228^{*}$ \\
$R_{3}$ & $\mathbf{2 . 8 0}$ & 2.81 & 1.27 & 1.70 & 0.19 & 0.60 & 0.10 & 0.36 & $236.898^{* * *}$ \\
\hline
\end{tabular}

${ }^{*} \mathrm{p}<.05,{ }^{* *} \mathrm{p}<.01,{ }^{* * *} \mathrm{p}<.001$ 
Finalmente, las categorías menos frecuentes son Exploración, Diversión, Trascendencia y Posesiones. Las tres primeras presentan diferencias significativas por grupos de edad, como se puede ver en el Cuadro 4. En Exploración (E) es el grupo de adolescentes el que se diferencia de los demás por la escasez de este tipo de contenidos. En Diversión (L) se observa una tendencia a incrementarse con la edad, lo mismo que con Trascendencia (T) aunque, en general, es la categoría menos frecuente.

Cuadro 4

Diferencias significativas en Exploración, Diversión y Trascendencia por grupo de edad

\begin{tabular}{lcccccccccc}
\hline Categoría & \multicolumn{2}{c}{$16-18$} & \multicolumn{2}{c}{$22-25$} & \multicolumn{2}{c}{$40-45$} & \multicolumn{2}{c}{$60-65$} & \multirow{2}{*}{$K-W$} \\
\cline { 2 - 8 } & $M$ & $D E$ & $M$ & $D E$ & $M$ & $D E$ & $M$ & $D E$ & \\
\hline Exploración & $\mathbf{0 . 2 8}$ & 0.68 & 0.70 & 1.00 & 0.64 & 0.88 & 0.58 & 1.10 & $24.281^{* * *}$ \\
Diversión & 0.71 & 1.07 & 0.82 & 1.34 & 0.94 & 1.25 & $\mathbf{1 . 2 6}$ & 1.56 & $13.509^{* *}$ \\
Trascenden. & 0.08 & 0.36 & 0.16 & 0.66 & 0.29 & 0.89 & $\mathbf{0 . 3 2}$ & 0.86 & $17.203^{* *}$ \\
\hline
\end{tabular}

${ }^{* *} \mathrm{p}<.01,{ }^{* * *} \mathrm{p}<.001$

Extensión de las metas

En el grupo en conjunto se observa un predominio de las metas localizadas en el Presente Abierto (54\%), seguido por el Futuro Distante $(24 \%)$. Las metas localizadas en el Pasado alcanzan escasamente el $1 \%$ del total. En la comparación por grupos de edad, se encuentran diferencias significativas en las tres categorías, Futuro Cercano, Futuro Distante y Presente Abierto ( $\mathrm{p}<.001$ ), como se puede ver en la Figura 2. Aquí se observa que las metas localizadas en un Futuro Distante tienden a disminuir conforme avanza la edad; lo contrario sucede con Presente Abierto que tiende 
Perspectiva temporal futura y satisfacción con la vida a lo largo del ciclo vital

a incrementarse con la edad. En Futuro Cercano la diferencia más saltante se encuentra en el grupo de los adultos tardíos quienes presentan un incremento de esta categoría temporal.

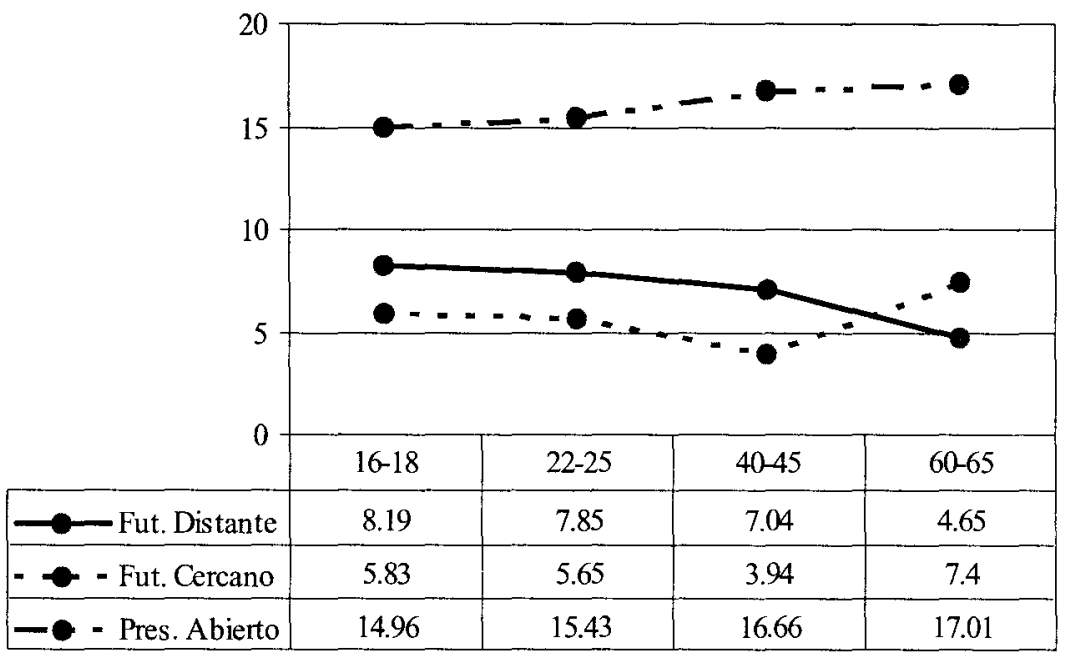

Figura 2. Extensión de las metas de acuerdo al grupo de edad.

De manera adicional, es posible determinar la extensión de la perspectiva temporal futura calculando la cantidad de metas ubicadas en el Futuro Cercano, frente a la cantidad de metas localizadas en el Futuro Distante. Cuanto menor es la proporción, la perspectiva temporal futura es más larga (Lens, 1998). Así, encontramos diferencias significativas en la extensión de la PTF de acuerdo a los grupos de edad $(\mathrm{K}-\mathrm{W}=46.438, g l=3, p<.001)$, donde los adultos tardíos son los que presentan la PTF más corta $(M=2.62$, $D E=2.54)$ y los adolescentes la más larga $(M=1.17, D E=1.32)$.

\section{Actitudes temporales}

Un primer dato importante se encuentra en el hecho de que, en promedio, las actitudes hacia el pasado, presente y futuro tienden a 
ser positivas en el grupo en conjunto (puntajes superiores a 4.5). Asimismo, resulta significativo que de las tres, la actitud hacia el futuro es la que presenta una media más alta $(M=5.5, \mathrm{DE}=0.74)$, en comparación con la actitud hacia el pasado $(M=5.09, \mathrm{DE}=0.69)$ y hacia el presente $(M=5.15, \mathrm{DE}=0.63)$. Aquí, es importante mencionar que las tres formas del TAS presentan correlaciones significativas $(p<.001)$ entre sí. La más alta se encuentra entre la forma presente y futuro ( $r=.645)$, luego entre la forma presente y pasada ( $r=.423$ ) y, finalmente, entre la forma futuro y pasado $(r=.267)$.

En la comparación por grupos de edad (Figura 3), encontramos diferencias significativas en la versión para el futuro y para el pasado. Aquí se puede ver, por un lado, que la actitud hacia el pasado tiende a hacerse más positiva conforme avanza la edad y que la actitud hacia el futuro se incrementa en los adultos jóvenes y luego tiende a disminuir. Por otro lado, se observa también que la diferencia entre la actitud hacia el pasado, presente y futuro tiende a unificarse con la edad.

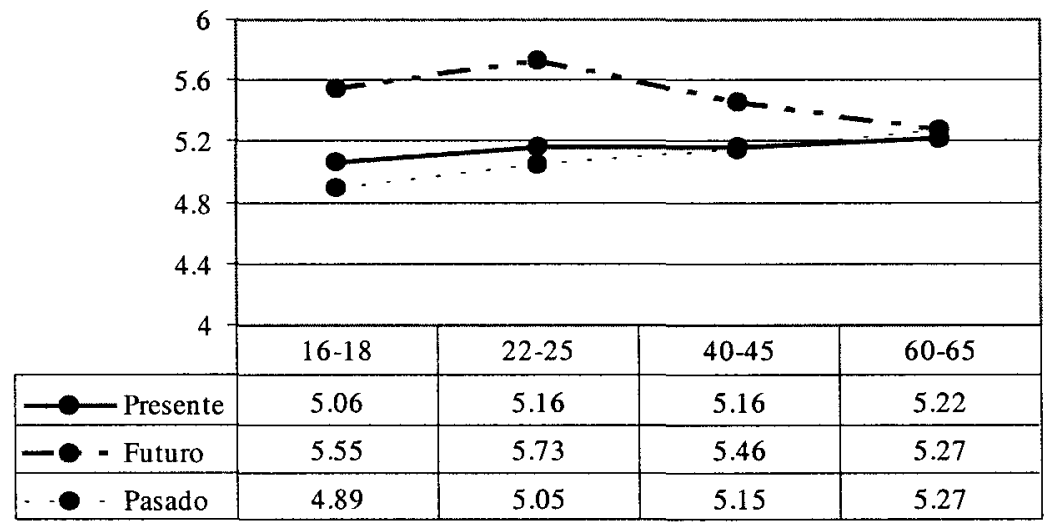

Figura 3. TAS de acuerdo a grupos de edad. 
Perspectiva temporal futura y satisfacción con la vida a lo largo del ciclo vital

\section{Satisfacción con la vida}

En el grupo en conjunto se encuentra que el $61.2 \%$ se encuentra entre satisfecho y muy satisfecho con su vida. En la comparación por grupos de edad, se puede observar en primer lugar que todos los grupos se encuentran por encima del punto neutral (20), lo que supone que, en general, en todos los grupos predomina el sentimiento de satisfacción con la vida. Sin embargo, se presentan diferencias importantes $(K-W=19.910$, $p<.001)$. Aquí se puede ver que los más satisfechos son los adultos tardíos y los menos satisfechos son los adolescentes (Figura 4).

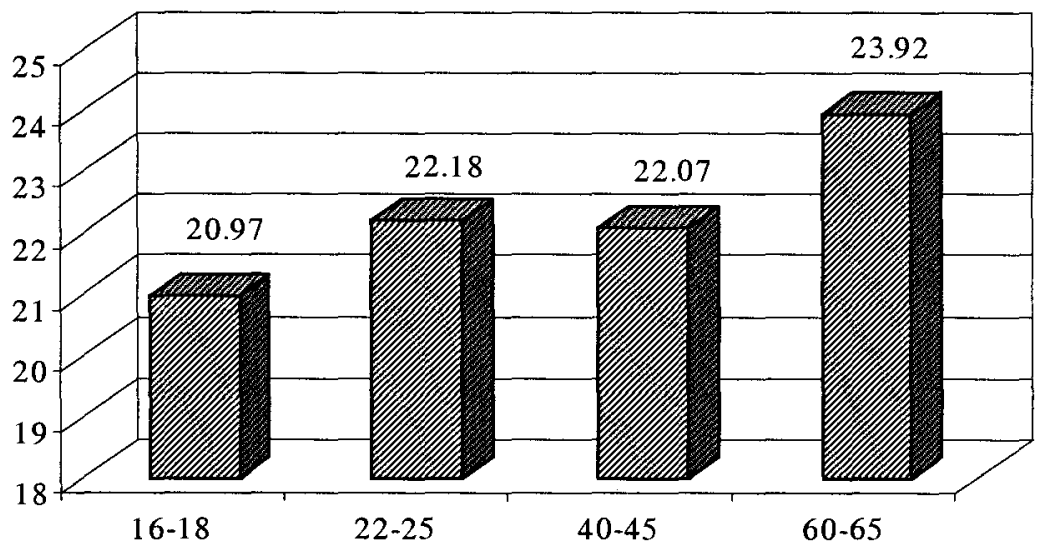

Figura 4: Satisfacción con la vida de acuerdo a grupo de edad.

\section{Sexo y nivel socioeconómico}

La variable sexo no muestra mayores diferencias en las diferentes variables estudiadas. Sólo encontramos una diferencia en la categoría de Contactos Interpersonales $(U=35894, p<.05)$ a 
favor de las mujeres $(M=11.02, D E=4.77)$ frente a los hombres $(M=10.06, D E=4.80)$.

La variable que sí marca importantes diferencias es el nivel socioeconómico (Cuadro 6). Así, en el NSE alto se incrementan las respuestas de Autorrealización y de Exploración. La actitud hacia el presente es más positiva y los niveles de satisfacción con la vida son más altos. En el NSE bajo aparece un predominio de respuestas de Posesiones materiales y mayor cantidad de metas ubicadas en el Futuro cercano.

\section{Cuadro 5}

Diferencias significativas de acuerdo al NSE

\begin{tabular}{lrrrrr}
\hline & \multicolumn{2}{c}{ NSE Alto } & \multicolumn{2}{c}{ NSE Bajo } & \multirow{2}{*}{$U$} \\
\cline { 2 - 5 } & $M$ & $D E$ & $M$ & \multicolumn{1}{c}{$D E$} & \\
\hline Autorrealización & $\mathbf{5 . 7 3}$ & 3.61 & 4.76 & 3.55 & $33964^{* *}$ \\
Exploración & $\mathbf{0 . 6 5}$ & 1.03 & 0.46 & 0.84 & $36492^{*}$ \\
Posesiones & 0.34 & 0.74 & $\mathbf{0 . 5 5}$ & 1.03 & $36314^{* *}$ \\
Futuro Cercano & 5.66 & 3.87 & $\mathbf{6 . 4 7}$ & 4.35 & $36680^{*}$ \\
TAS Presente & $\mathbf{5 . 2 3}$ & 0.61 & 5.07 & 0.62 & $35460.5^{* *}$ \\
Satisfacción & $\mathbf{2 3 . 3 5}$ & 6.41 & 21.21 & 6.31 & $32910^{* * *}$ \\
\hline
\end{tabular}

$* p<.05, * * p<.01, * * * p<.001$

\section{Correlaciones}

En primer lugar analizamos las correlaciones entre las metas del MIM y la ubicación temporal de las mismas. Aquí encontramos que existen correlaciones en todas las variables de contenido con excepción de Exploración. La correlación más alta se encuentra en Autorrealización y Futuro Distante $(r=748)$. Las metas sobre Sí Mismo correlacionan básicamente con Presente Abierto ( $r=.475$ ), las de Realización con Futuro Cercano $(r=.491)$, Contacto con Presente Abierto ( $r=.302$ ), Diversión con Futuro 
Perspectiva temporal futura y satisfacción con la vida a lo largo del ciclo vital

Cercano ( $r=.453$ ), Trascendencia con Presente Abierto $(r=.229)$ y Posesiones con Futuro Cercano. $(r=.263)$, todas ellas con un $p<.0001$.

Entre las metas del MIM con las actitudes temporales sólo se encuentran correlaciones pequeñas pero significativas. Así, encontramos una correlación positiva entre Autorrealización y actitud hacia el futuro $(r=.142, p<.01)$, otra negativa entre Diversión y también actitud hacia el futuro $(r=-.118, p<.01)$, y otra más pequeña, positiva, entre las actitudes hacia el pasado y las metas de Contacto Interpersonal $(r=.102, p<.05)$. Algo similar ocurre al correlacionar las metas del MIM con el SWLS, también aquí las correlaciones son pequeñas pero significativas. Encontramos una correlación negativa ( $r=-.149$, $p<.001)$ entre Satisfacción y metas sobre Sí Mismo, y otra positiva entre Satisfacción y Contacto Interpersonal ( $r=.123$, $p<.01$ ).

Por otro lado analizamos las correlaciones de la ubicación temporal de las metas y las actitudes temporales. Aquí sólo encontramos una correlación pequeña y positiva entre Futuro Distante y actitud hacia el futuro $(r=.102, p<.05)$ y una correlación negativa también pequeña entre Futuro Distante y actitud hacia el pasado ( $r=-.103, p<.05)$. Lo mismo sucede al correlacionar la extensión temporal de las metas y la satisfacción con la vida. Sólo aparece una correlación pequeña y negativa entre Satisfacción y metas ubicadas en el Pasado ( $r=-.104$, $p<.05)$.

Finalmente analizamos la correlación entre el nivel de satisfacción y las actitudes temporales. Aquí aparecen correlaciones medias en las tres formas del TAS. Con el TAS Presente $(r=.439)$, con el TAS Futuro $(r=.229)$ y con el TAS Pasado ( $r=.253$ ), todas ellas con un $p<.001$. 


\section{Análisis de conglomerados}

Con el fin de examinar si los participantes de la investigación se agrupan de determinada forma en base a las variables de la investigación, optamos por realizar un análisis de clusters, en el que obtuvimos tres grupos claramente definidos. Las variables que mostraron mayor poder de discriminación en base a un análisis de varianza (ANOVA) fueron: satisfacción con la vida, actitud hacia el pasado, presente y futuro, metas de Autorrealización y Contacto Interpersonal y, finalmente, localización de metas en el Futuro Distante y Futuro Cercano.

Lo más característico de cada uno de los clusters fue:

- Cluster 1: menor satisfacción con la vida y actitud menos positiva ante el Presente y Futuro.

- Cluster 2: mayor satisfacción con la vida y actitud más positiva ante el Presente, Pasado y Futuro, además de mayor cantidad de metas de Contacto Interpersonal.

- Cluster 3: mayor Autorrealización y menor Contacto Interpersonal y PTF más larga (mayor cantidad de metas en Futuro Distante y menor en Futuro Cercano).

Con el fin de identificar a los participantes de cada uno de los clusters, aplicamos la prueba de chi cuadrado para las tres variables centrales del estudio: grupo de edad, sexo y nivel socioeconómico. Encontramos diferencias significativas de acuerdo a los grupos de edad y de acuerdo al NSE (Cuadro 6). El sexo no presenta diferencias entre los clusters. 
Perspectiva temporal futura y satisfacción con la vida a lo largo del ciclo vital

Cuadro 6

Clusters de acuerdo a grupo de edad y NSE

\begin{tabular}{lrrrrrrr}
\hline & & \multicolumn{2}{c}{1} & \multicolumn{2}{c}{2} & \multicolumn{2}{c}{3} \\
\cline { 3 - 8 } & & $f$ & $\%$ & \multicolumn{1}{c}{$f$} & $\%$ & $f$ & $\%$ \\
\hline \multirow{2}{*}{ Edad } & $16-18$ & 49 & $22.7 \%$ & 22 & $15.5 \%$ & $\mathbf{7 2}$ & $\mathbf{3 4 . 0 \%}$ \\
$X^{2}=39.128$ & $22-25$ & 41 & $19.9 \%$ & 33 & $23.2 /$ & 68 & $32.1 \%$ \\
$p<.001$ & $40-45$ & 58 & $26.9 \%$ & 45 & $31.7 \%$ & 40 & $18.9 \%$ \\
& $60-65$ & 68 & $31.5 \%$ & 42 & $29.6 \%$ & 32 & $15.1 \%$ \\
& $22-25$ & 41 & $100 \%$ & 142 & $100 \%$ & 212 & $100 \%$ \\
\hline \multirow{2}{*}{ NSE } & Alto & 103 & $47.7 \%$ & $\mathbf{8 5}$ & $\mathbf{5 9 . 9 \%}$ & $\mathbf{9 7}$ & $\mathbf{4 5 . 8 \%}$ \\
$X^{2}=7.512$ & Bajo & 113 & $52.3 \%$ & 57 & $40.1 \%$ & $\mathbf{1 1 5}$ & $\mathbf{5 4 . 2 \%}$ \\
$p<.05$ & Total & 216 & $100 \%$ & 142 & $100 \%$ & 212 & $100 \%$ \\
\hline
\end{tabular}

Aquí puede verse que el cluster 1 (en general, menos positivos frente a la vida) predomina en los adultos tardíos, el cluster 2 (más positivos y más interesados en el Contacto Interpersonal) en los adultos medios y el cluster 3 (con una PTF más larga y mayor énfasis en la Autorrealización) en los adolescentes y adultos jóvenes. Asimismo, los clusters 1 y 3 destacan en los participantes de NSE bajo y el cluster 2 en los de NSE alto.

\section{Discusión}

En primer lugar, en el grupo en general encontramos que, independientemente de la edad, el sexo o el nivel socioeconómico, el grupo concentra la mayor parte de sus metas en Contacto Interpersonal, Sí Mismo y Autorrealización. Estos resultados confirman la importancia de los vínculos interpersonales a lo largo del desarrollo, el tema de identidad como eje de la personalidad y la necesidad de autorrealización como una necesidad fundamental a lo largo de la vida, lo que va en el mismo sentido a los planteamientos de Nuttin (1985) en torno a las dos necesidades 
básicas del ser humano, la orientación hacia el autodesarrollo y hacia los demás.

Existe, asimismo, una tendencia a ubicar las metas predominantemente en el presente abierto, que es la categoría que presenta las correlaciones más altas con las metas de Sí Mismo y Contacto Interpersonal. Las actitudes hacia el pasado, presente y futuro se encuentran positivamente correlacionadas; en el grupo en general tienden a ser positivas, siendo la actitud hacia el futuro la más alta de las tres, lo que hace referencia a una actitud predominantemente optimista frente a la vida y al futuro en particular. Asimismo, y significativamente correlacionado con lo anterior, en el grupo predomina la satisfacción con la vida. Estos resultados confirman así la correlación entre satisfacción y actitud optimista frente a la vida, encontrada en otros estudios con poblaciones normales (Chang \& Sanna, 2003; Díaz Morales \& Sánchez López, 2002). Cabe mencionar además que el grupo de participantes de esta investigación es relativamente homogéneo pues todas las personas se encuentran realizando algún tipo de actividad productiva (los más jóvenes estudiando y los mayores trabajando), lo que constituye en el Perú ya un elemento de diferenciación.

Analizaremos a continuación las principales características de cada uno de los grupos de edad, de tal manera que podamos observar cómo van cambiando las diferentes variables estudiadas a lo largo del ciclo vital, desde la adolescencia hasta la adultez tardía.

Los adolescentes presentan una elevada cantidad de metas relacionadas al Contacto Íntimo y a la Pareja, esto se puede asociar al florecimiento de la sexualidad, a los inicios de la búsqueda de intimidad y también a una suerte de confirmación de la propia identidad sexual. También son elevadas las metas relativas a Padres y Amigos, que estarían expresando la importancia de la familia y los pares como grupos de referencia fundamentales en esta etapa. Asimismo, se encuentra una elevación de las metas relacionadas al 
Perspectiva temporal futura y satisfacción con la vida a lo largo del ciclo vital

Autoconcepto y la Autonomía, que tienen que ver con la tarea central de esta etapa, el logro de la identidad, donde la definición de sí mismo y la necesidad de independencia adquieren un papel central (Castillo, 1999; Erikson, 1993; Rice, 2000).

Las metas de Autorrealización son también elevadas, particularmente la autorrealización en general, que supone respuestas vagas que expresan únicamente el deseo de triunfar o tener éxito. Este dato es muy similar a lo que encuentra Herrera (2002) en su investigación con adolescentes a través del MIM y puede asociarse a la tendencia a la idealización en la adolescencia, donde es frecuente que se expresen aspiraciones generales en lugar de proyectos más concretos y reales (Guichard, 1995; Rice, 2000). Esto se confirma además con la escasez de metas sobre Exploración, las cuales se presentan en este grupo por debajo de los otros. La exploración sería un aspecto fundamental para el desarrollo de planes y proyectos, su ausencia corrobora la presencia de proyectos más fantasiosos y menos basados en la realidad. En la misma línea, las metas de Realización son también elevadas y en particular todo lo referente a Estudios, que constituye el mundo más inmediato del adolescente.

En cuanto a las categorías temporales se encuentra una elevación en la cantidad de metas localizadas en el Futuro Distante, así como una PTF más larga. Esto podría estar indicando cierta orientación hacia el futuro que se corrobora con los resultados del TAS donde se encuentra una actitud muy positiva hacia el futuro, bastante distante del presente y una actitud menos positiva hacia el pasado. Esto coincide con otros estudios que refieren una PTF más larga en los adolescentes y una orientación hacia el futuro (Nurmi, Poole \& Seginer, 1992, 1995). Sin embargo, esto podría entenderse también como cierta idealización del futuro como un espacio de realización más bien mágico, si tomamos en cuenta la distancia entre la actitud hacia el futuro y hacia el presente, la cantidad de metas de Autorrealización en general y la escasez de metas de Exploración. 
Finalmente, el grupo de adolescentes es el que presenta menores niveles de satisfacción con la vida en comparación con los otros grupos de edad, lo cual podría asociarse con lo que Castillo (1999) describe como el inconformismo adolescente asociado a una suerte de rebeldía necesaria para su desarrollo.

El grupo de adultos jóvenes presenta un buen número de semejanzas con el grupo de adolescentes. Esto probablemente tenga que ver con que los rangos de edad son bastante cercanos (16 a 18 y 22 a 25$)$ y, a diferencia de lo que sucede en otros países, los adultos jóvenes de este rango de edad viven casi en su totalidad en el hogar de sus padres, manteniendo aún vínculos de dependencia con ellos, los que probablemente influyen en las similitudes encontradas. Estas semejanzas podrían asociarse también a lo que Castillo (1999) describe como el fenómeno de la adolescencia ampliada, relacionado a un retraso en la emancipación de los jóvenes, quienes retrasan cada vez más su salida del hogar familiar. En un país como el Perú este retraso tiene que ver además con características propias del contexto, como las altas tasas de desempleo y subempleo que no facilitan este proceso de independización.

Así, de manera similar a los adolescentes, el grupo de adultos jóvenes presenta una elevación en las metas de Contacto Intimo y Pareja, en esta etapa más asociadas al logro de la intimidad, aspecto central de acuerdo a Erikson (1993). Un dato significativo aquí se encuentra en la elevación de metas sobre Contacto Egocéntrico, que si bien no es especialmente alta, sí es significativamente más alta que en los otros grupos de edad. Esto podría estar indicando que el grupo aún se encuentra muy centrado en sí mismo; si bien hay un interés claro por relacionarse, se trata de un interés que responde en parte aún a fines más egocéntricos, es la necesidad de otro que confirme o refuerce su propia identidad.

En este mismo sentido, es el grupo que presenta mayor cantidad de metas de Sí Mismo, que confirmaría lo anterior, ya 
Perspectiva temporal futura y satisfacción con la vida a lo largo del ciclo vital

que sería un grupo que estaría centrado aún en una tarea propia de la etapa anterior, es decir, la consolidación de la identidad. La subcategoría de Autoconcepto también elevada, va en este mismo sentido. Se trataría así de un grupo que aún está buscando resolver aspectos propios de su identidad. La correlación negativa encontrada entre las metas sobre Sí Mismo y satisfacción con la vida va en el mismo sentido de lo descrito por Salmela y Nurmi (1997), quienes refieren menores niveles de bienestar psicológico en aquellos que presentan más metas sobre Sí Mismo, porque esto supone aspectos aun no resueltos que serían una fuente de malestar. Recordemos que se trata de un grupo que recién está entrando a la adultez y resulta hasta cierto punto esperable que mantenga aún aspectos propios de una etapa anterior.

La elevación de metas en la subcategoría de Autonomía estaría expresando la necesidad de independencia característica de los adultos jóvenes. De manera similar al grupo de adolescentes, las metas de Autorrealización y Realización son altas; la diferencia está en una mayor tendencia a la Autorrealización profesional, la cual podría asociarse con el momento en el que se encuentran, es decir, la culminación de una profesión, esto hace que las respuestas sean menos vagas que en los adolescentes y que la profesión sea sentida como el principal medio de autorrealización.

En cuanto a extensión, presentan mayor cantidad de metas en un Futuro Distante, al igual que el grupo adolescente, y una larga PTF, que estaría indicando que se mantiene la orientación general hacia el futuro en este grupo. Asimismo, es el grupo que presenta una actitud hacia el futuro más positiva con una importante distancia con el presente y pasado, lo que nuevamente podría estar haciendo referencia a cierta idealización del futuro, que se confirma también en el estudio de Staudinger et al. (2003), quienes encuentran un incremento en los adultos jóvenes en el bienestar futuro en comparación con el pasado y presente. 
Por otro lado, se presenta un incremento de los niveles de satisfacción con la vida a diferencia de los adolescentes. Esto podría entenderse desde la sensación de haber cumplido ya con algunas de sus metas de vida, al encontrarse terminando una carrera universitaria, que fue uno de los principales objetivos de la etapa anterior.

El ingreso a la adultez media sí supone cambios importantes de la etapa anterior. Es un grupo que se caracteriza por presentar mayor cantidad de metas de Contacto Interpersonal y de las subcategorías de Contacto para Otros y para la Humanidad, así como mayor cantidad de metas vinculadas a la Sociedad. Esto se relaciona con un mayor interés y preocupación por los otros y un claro rompimiento con el egocentrismo de la etapa anterior, que también se confirma con la elevación de metas sobre Trascendencia. Asimismo, el interés por la Familia y los Hijos aumenta significativamente con respecto a la etapa anterior. Esto coincide con los planteamientos de Erikson (1993), Neugarten (1999) y Nurmi (1994), quienes hacen referencia a relaciones más cercanas en esta etapa.

De otro lado, se encuentra una disminución de metas sobre Sí Mismo, que confirma lo anterior, pero un incremento de metas sobre Preservación, que podría asociarse a la mayor preocupación por la salud correspondiente a los inicios de la senescencia. También se incrementan las metas relacionadas al desarrollo de aptitudes, asociado a una etapa de gran productividad. La disminución de metas sobre Autorrealización y Realización está relacionada al aumento de metas vinculadas a los Hijos, que en esta etapa se convierten en la principal fuente de autorrealización.

La mayor parte de las metas tiende a ubicarse en el Presente Abierto y se inicia una disminución de las metas localizadas en el Futuro Distante en relación a los adultos jóvenes, sin embargo se mantiene una larga PTF. Con respecto a las actitudes temporales, se observa que la actitud hacia el Pasado se hace más positiva y una ligera disminución de la actitud positiva hacia el Futuro, 
Perspectiva temporal futura y satisfacción con la vida a lo largo del ciclo vital

aunque sigue siendo la más alta. Esto probablemente tiene que ver con los inicios del cambio en la perspectiva temporal descrito por Lefrancois (2001), Neugarten (1999) y Stassen-Berger (2001), que supone una evaluación distinta del tiempo, probablemente con una menor idealización del futuro como en los adultos jóvenes. Asimismo, la distancia entre la actitud hacia el Presente y Futuro se hace más corta, por una suerte de mayor balance entre lo que se vive y lo que se tiene por vivir (Staudinger et al., 2003). Asimismo, los niveles de satisfacción con la vida se mantienen con respecto a los adultos jóvenes.

Los adultos tardíos presentan un buen numero de semejanzas con los adultos medios en contraposición con los grupos menores, que probablemente esté relacionada a las edades del grupo de adultos tardíos, ya que es un grupo que recién estaría ingresando a esta etapa y que corresponde a lo que Neugarten (1999) describe como un grupo de viejos-jóvenes. Asimismo, se podría hablar de mayor estabilidad en diferentes aspectos de la personalidad.

Se trata de un grupo en el que se mantiene la elevación de metas sobre Contacto Interpersonal, Contacto para Otros, para la Humanidad y en la Sociedad, que va en el mismo sentido de la adultez media. Lo mismo ocurre con las metas sobre Familia e Hijos. Al interior de la categoría de Sí Mismo existe un incremento de metas sobre Preservación, que supone una mayor preocupación por la salud en esta etapa con respecto a la etapa anterior, que corresponde también a lo descrito por Estaún (1996), quien se refiere a la salud como una de las mayores preocupaciones en esta etapa. Se mantiene la frecuencia de metas de Autorrealización, Realización y Trascendencia de la etapa anterior, y se presenta un importante incremento de metas sobre Diversión, que se puede interpretar como la presencia de mayores libertades y pasatiempos asociada a que parte de las tareas más trascendentes de la vida ya han sido cubiertas (Lefrancois, 2001).

Por otro lado, disminuye significativamente la cantidad de metas ubicadas en el Futuro Distante y aumentan las metas en el 
Futuro Cercano y Presente Abierto, la PTF se hace así más corta. La actitud hacia el Pasado se hace más positiva y la actitud hacia el Futuro se hace menos positiva con lo que las tres se ubican en un mismo nivel. Esto coincide con lo descrito por Staudinger et al. (2003), quienes se refieren a que las personas de esta etapa se reconcilian con su pasado y aceptan el futuro por lo que aparece una mayor congruencia entre los tres.

Finalmente, se trata del grupo que presenta mayores niveles de satisfacción con la vida. Esto se puede relacionar con los hallazgos de Lennings (2000), quien encuentra en los viejosjóvenes una alta correlación entre optimismo y satisfacción.

Hasta aquí podemos afirmar, siguiendo los planteamientos de Nurmi (1994), que las tareas de desarrollo están estrechamente relacionadas al tipo de metas que las personas describen. Vemos asimismo que los cambios más importantes en el ciclo vital se presentan alrededor de los 40 años $o$ al ingresar a la adultez media.

La escasez de diferencias en las variables estudiadas con respecto a la variable sexo, nos lleva a suponer que se trata de procesos comunes a ambos sexos. La única categoría que presenta diferencias es la de Contacto Interpersonal, más presente en las mujeres que va en el mismo sentido de lo descrito por los teóricos del desarrollo. El nivel socioeconómico sí resulta una variable fundamental a tomarse en cuenta en los estudios sobre PTF. Estas diferencias dan cuenta de lo que en el Perú significa pertenecer a un NSE bajo, donde las carencias afectan a todos los aspectos de la vida.

Del mismo modo, las asociaciones entre satisfacción con la vida y PTF dan cuenta de constructos estrechamente ligados entre sí. Las correlaciones más significativas se presentan entre satisfacción con la vida y actitudes temporales y especialmente con la actitud hacia el presente.

Por último, el análisis de los conglomerados nos ha permitido asociar todas las variables del estudio e identificar tres grupos 
Perspectiva temporal futura y satisfacción con la vida a lo largo del ciclo vital

claramente definidos. Los menos positivos frente a la vida (o más realistas) con relación a los grupos de edad son predominantemente los adultos tardíos, los más positivos frente a la vida y más interesados en el contacto interpersonal son predominantemente los adultos medios y el grupo de NSE alto, y los más centrados en el futuro, con mayores necesidades de autorrealización, se localizan esencialmente en los grupos de adolescentes y adultos jóvenes, así como en los participantes de NSE bajo.

La presente investigación nos permite remarcar la utilidad del modelo de Nuttin para la evaluación de la PTF. La coherencia del modelo se expresa, por un lado, en las correlaciones entre los instrumentos utilizados $\mathrm{y}$, por otro, en la consistencia de los mismos. Asimismo, se expresa en las semejanzas entre nuestros resultados y los de otras investigaciones, incluso con modelos distintos.

\section{Referencias}

Apoyo. (2002). Niveles socioeconómicos de la Gran Lima. Lima: Apoyo.

Bouffard, L., Bastin, E. \& Lapierre, S. (1994). The personal future in old age. En Z. Zaleski (Ed.), Psychology of future orientation (pp. 75-94). Lublin: Towarzystwo Naukowe KUL. Castillo, G. (1999). El adolescente y sus retos. Madrid: Pirámide. Chang, E. \& Sanna, L. (2003). Optimism, accumulated life stress, and psychological and physical adjustment: Is it always adaptive to expect the best? Journal of Social and Clinical Psychology, 22(1), 97-115.

Craig, G. (1994). Desarrollo psicológico. México: Prentice Hall Hispanoamericana.

De Volder, M. \& Lens, W. (1982). Academic achievement and future time perspective as a cognitive-motivational concept. 
Patricia Martínez U.

Journal of Personality and Social Psychology, 42(3), 566571.

Díaz Morales, J. \& Sánchez López, M. (2002). Metas personales

y satisfacción autopercibida. En G. Hernández (Ed.), Motivación animal y humana (pp. 359-380). Mexico: Manual Moderno.

Diener, E., Oishi, S. \& Lucas, R. (2003). Personality, culture and subjective well-being: Emotional and cognitive evaluations of life. Annual Review of Psychology, 54, 403-425.

Erikson, E. (1993). El ciclo vital completado. México: Paidós.

Estaún, S. (1996). La dimensión temporal en el anciano: la perspectiva temporal. En N. Sáez, R. Rubio \& A. Dosil (Eds.), Tratado de Psicogerontología (pp. 167-187). Valencia: Promolibro.

Frazier, L., Johnson, P., Gonzales, G. \& Kafka, C. (2002).

Psychosocial influences on possible selves: A comparison of three cohorts of older adults. International Journal of Behavioral Development, 26(4), 308-317.

Guichard, J. (1995). La escuela y las representaciones de futuro de los adolescentes. Barcelona: Alertes.

Hernández, R., Fernández, C. \& Baptista, P. (1998). Metodología de la investigación. México: McGraw Hill.

Herrera, D. (2002). Social insertion of high school graduates in Lima. Tesis doctoral no publicada, Universidad Católica de Lovaina, Bélgica.

Husman, J. \& Lens, W. (1999). The role of the future in student motivation. Educational Psychologist, 34(2), 113-125.

Lefrancois, G. (2001). El ciclo de la vida. México: International Thomson.

Lennings, C. (2000). Optimism, satisfaction and time perspective in the elderly. International Journal of Aging and Human Development, 51(3), 167-181.

Lens, W. \& Moreas, M. (1994). Future time perspective: Individual and societal approach. En Z. Zaleski (Ed.), 
Perspectiva temporal futura y satisfacción con la vida a lo largo del ciclo vital

Psychology of future orientation (pp. 23-38). Lublin: Towarzystwo Naukowe KUL.

Lens, W. (1998). El rol de la perspectiva de tiempo futuro en la motivación estudiantil. Persona, 1, 67-91.

Martínez, P. (2002). Perspectiva temporal futura en adolescentes de Lima-Perú. Monografía de investigación no publicada, Universidad Autónoma de Barcelona, España.

Neugarten, B. (1999). Los significados de la edad. Barcelona: Herder.

Nurmi, J. E. (1991). How do adolescents see their future? A review of the development of future orientation and planning. Developmental Review, 11, 1-59.

Nurmi, J. E. (1994). The development of future orientation in lifespan context. En Z. Zaleski (Ed.), Psychology of future orientation (pp. 63-74). Lublin: Towarzystwo Naukowe KUL. Nurmi, J. E., Poole, M. \& Seginer, R. (1992). Future hopes and fears questionnaire. Helsinki: University of Helsinki Department of Psychology.

Nurmi, J. E., Poole, M. \& Seginer, R. (1995). Tracks and transitions: A comparison of adolescent future oriented goals, explorations, and commitments in Australia, Israel, and Finland. International Journal of Psychology, 30(3), 355-375.

Nuttin, J. (1982). Teoría de la motivación humana. Barcelona: Paidós.

Nuttin, J. (1985). Future time perspective and motivation. Lovaina: Leuven University Press \& Lawrence Erlbaum.

Pavot, W. \& Diener, E. (1993). Review of the Satisfaction with Life Scale. Psychological Assessment, 5(2), 164-172.

Rice, P. (2000). Adolescencia: desarrollo, relaciones y cultura. Madrid: Prentice Hall.

Salmela, K. \& Nurmi, J. E. (1997). Goal contents, well being and life context during transition to university: A longitudinal study. International Journal of Behavioral Development, 20(3), 471-491. 
Stassen-Berger, K. (2001). Psicologia del desarrollo: adultez y vejez. Madrid: Médica Panamericana.

Staudinger, U., Bluck, S. \& Herzberg, P. (2003). Looking back and looking ahead: Adult age differences in consistency of diachronous ratings of subjective well-being. Psychology and Aging, 18(1), 13-24.

Van Calster, K., Lens, W. \& Nuttin, J. (1987). Affective attitude toward the personal future: Impact on motivation in high school boys. American Journal of Psychology, 100(1), 1-13.

Zaleski, Z. (1994). Towards a psychology of the personal future. En Z. Zaleski (Ed.), Psychology of future orientation (pp. 1020). Lublin: Towarzystwo Naukowe KUL.

Zaleski, Z., Chlewinski, Z. \& Lens, W. (1994). Importance of and optimism-pessimism in predicting solution to world problems: An intercultural study. En Z. Zaleski (Ed.), Psychology of future orientation (pp. 207-228). Lublin: Towarzystwo Naukowe KUL. 